\title{
Direct targeting of SUZ12/ROCK2 by miR-200b/c inhibits cholangiocarcinoma tumourigenesis and metastasis
}

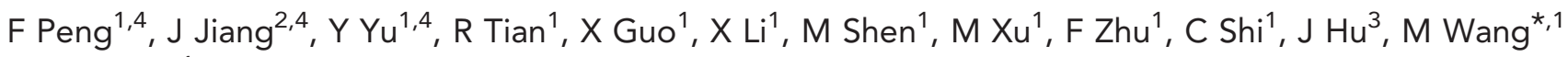 \\ and R Qin ${ }^{*} 1$
}

${ }^{1}$ Department of Biliary-Pancreatic Surgery, Affiliated Tongji Hospital, Tongji Medical College, Huazhong University of Science and Technology, 1095 Jiefang Avenue, Wuhan, Hubei 430030, China; ${ }^{2}$ Department of Biliary-Hepatic Surgery, Affiliated Hospital of Guiyang Medical College, Guiyang, Guizhou, China and ${ }^{3}$ Department of Colon Cancer, Tianjin Medical University Cancer Institute and Hospital, Tianjin, China

Background: The multidrug resistance and distant metastasis of cholangiocarcinoma result in high postoperative recurrence and low long-term survival rates. It has been demonstrated that the ectopic expression of miR-200 suppresses the multidrug resistance and metastasis of cancer. However, the expression and function of miR-200 in cholangiocarcinoma has not yet been described.

Methods: In this study, we identified dysregulated microRNAs (miRNAs, miR) in cholangiocarcinoma tissue by microarray analysis, and subsequent real-time PCR and northern blot analyses validated the expression of candidate miR. We performed functional analyses and investigated the relationship between miR-200b/c expression and the properties of cholangiocarcinoma cells. A dual luciferase assay was applied to examine the effect of miRNAs on the $3^{\prime}$-UTR of target genes, and we demonstrated the function of the target gene by siRNA transfection identifying the downstream pathway via western blotting.

Results: We found significantly downregulated expression of four miR-200 family members (miR-200a/b/c/429) and then confirmed that ectopic miR-200b/200c inhibits the migration and invasion of cholangiocarcinoma cells both in vitro and in vivo. We found that miR-200b/c influenced the tumourigenesis of cholangiocarcinoma cells including their tumour-initiating capacity, sphere formation, and drug resistance. We further found that miR-200b/c regulated migration and invasion capacities by directly targeting rho-kinase 2 and regulated tumorigenic properties by directly targeting SUZ12 (a subunit of a polycomb repressor complex).

Conclusion: Our study shows that miR-200b/c has a critical role in the regulation of the tumorigenic and metastatic capacity of cholangiocarcinoma and reveals the probable underlying mechanisms.

Cholangiocarcinoma is generally acknowledged as being difficult to diagnose and treat. Despite improvements in treatment, the survival of patients remains poor (Khan et al, 2008), which is attributed to early extensive local tumour invasion and distant metastasis, as well as multichemoresistance (Khan et al, 2005; Blechacz and Gores, 2008). The development of cholangiocarcinoma is a multistep process, with an accumulation of genetic and epigenetic alterations (Evert et al, 2013; Timmer et al, 2013). Therefore, a better understanding of the molecular mechanisms of the multidrug resistance, distant metastasis, and tumorigenic properties of cholangiocarcinoma is likely to lead to novel therapeutic strategies for cholangiocarcinoma (Visvader and Lindeman, 2008).

\footnotetext{
*Correspondence: Professor R Qin or Dr M Wang; E-mails: ryqin@tjh.tjmu.edu.cn or tjwangmin0013128@gmail.com

${ }^{4}$ These authors contributed equally to this work
}

Received 16 June 2013; revised 5 September 2013; accepted 1 October 2013; published online 29 October 2013 
MicroRNAs (miRNAs) are a well-known class of small RNAs that inhibit gene expression at the post-transcriptional level by directly targeting regions of sequence complementarity in the $3^{\prime}$-untranslated regions ( $3^{\prime}$-UTRs) of mRNAs (He and Hannon, 2004). The miR-200 family, including the miR-200a/141 and miR$200 \mathrm{~b} / \mathrm{c} / 429$ clusters, is involved in neurogenesis (Karres et al, 2007), the regulation of embryonic and adult stem cells, and cancer stem cells (CSCs) (Shimono et al, 2009; Wellner et al, 2009; Samavarchi-Tehrani et al, 2010), chemosensitivity, and apoptosis (Cochrane et al, 2010; Schickel et al, 2010). The miR-200 family also influences the epithelial-to-mesenchymal transition (EMT) through the Zeb-E-cadherin axis (Burk et al, 2008; Korpal et al, 2008; Park et al, 2008). However, recent studies have yielded conflicting results in terms of the tumour suppressor function of this family (Dykxhoorn et al, 2009), it remains unclear whether the metastasis suppressor functions of the miR-200 family are mediated entirely or only partially through the Zeb-E-cadherin axis (Korpal et al, 2011). Indeed, although the potential of other cancer-related functions of the miR-200 family warrants further research, few studies on the relationship between the expression levels of miRNAs and cholangiocarcinoma cells have been published. Therefore, we conducted miRNA profiling in cholangiocarcinoma tissues and found a differential expression of miRNAs, including four of the five miR-200 family members, which have been shown to have an important role in regulating the properties of metastasis and invasion. Nonetheless, the function of miR-200 in cholangiocarcinoma tumourigenesis has not been clearly elucidated.

Here, we demonstrate that $\mathrm{miR}-200 \mathrm{~b} / \mathrm{c}$ regulates the metastatic capacity and tumorigenic properties of cholangiocarcinoma, including tumour-initiating capacity, sphere formation, and drug resistance. We also show that $\mathrm{miR}-200 \mathrm{~b} / \mathrm{c}$ inhibits invasion and migration by directly targeting other genes such as rho-kinase 2 (ROCK2). Moreover, miR-200b/c influences cholangiocarcinoma tumourigenesis by silencing SUZ12 (a subunit of a polycomb repressor complex). These results provide insight into the molecular mechanisms of cholangiocarcinoma regulation and may suggest a novel therapeutic strategy for cholangiocarcinoma treatment.

\section{MATERIALS AND METHODS}

Tumours. Human cholangiocarcinoma tissues were obtained from 14 patients with postoperative pathological diagnosed perihilar or distal biliary cholangiocarcinoma from September 2010 to May 2011 at the Department of Biliary-Pancreatic Surgery, Affiliated Tongji Hospital (Hubei, China) (Supplementary Table 1). Human sample collection procedures were approved by the China Ethical Review Committee.

MiRNA microarrays. Total RNA was extracted from three cholangiocarcinoma tissues and their corresponding normal bile ducts using a mirVana miRNA Isolation Kit (Ambion, Austin, TX, USA) according to the manufacturer's instructions. An Agilent Human miRNA Microarray Kit (V2) (Agilent Inc, Santa Clara, CA, USA) (Sanger database v.10.1) was used for analysis. RNA labelling and hybridisation on Agilent miRNA microarray chips were performed with an miRNA Labelling Reagent and Hybridization Kit (Agilent Technologies). Microarray data analyses including GO analysis, Pathway Analysis, Path-net, Signal-net, and MicroRNA-gene network were performed (Genminix Company, Shanghai, China). The microarray data were deposited in NCBI's Gene Expression Omnibus (GEO) public database (http://www.ncbi.nlm.nih.gov/geo/; Gene Expression Omnibus accession number, GSE47764).
Cell culture and transfections. The TFK-1 cell line was purchased from DSMZ by Professor Shengquan Zou of the Affiliated Tongji Hospital. The QBC939 cell line was provided by Professor Shu-guang Wang of The Third Military Medical University, Chongqing, China. All cell lines were cultured in RPMI-1640 medium supplemented with $10 \%$ fetal bovine serum (FBS), L-glutamine, and $1 \%$ penicillin/streptomycin at $37^{\circ} \mathrm{C}$ in a $5 \%$ $\mathrm{CO}_{2}$ incubator. Cells were seeded in 6-well plates and transfected with $\mathrm{miR}-200 \mathrm{~b} / \mathrm{c}$ mimics/antagomir or negative control (NC) (Ambion) at a final concentration of $50 \mathrm{~nm}$ using siPORT NeoFX Transfection Agent (Ambion). Small interfering RNA and the NC were purchased from RiboBio (Guangzhou, China), The sequences of siRNA could be found in Supplementary Table 4. MiRNA-200b/ c overexpression and knockdown lentiviruses, sh-SUZ12 and sh-ROCK2, were constructed by and purchased from Genechem (Shanghai, China). All transfections were carried out according to the manufacturer's instructions.

RNA extraction and RT-qPCR. Total RNA was isolated as described above. Real-time polymerase chain reaction was carried out using $50 \mathrm{ng}$ or $500 \mathrm{ng}$ of total RNA using a TaqMan MicroRNA Reverse Transcription Kit (Applied Biosystems, Carlsbad, CA, USA) or PrimeScript Reverse Transcription Kit (TAKARA, Dalian, China) for miRNA and mRNA analyses, respectively. Real-time fluorescence quantitative polymerase chain reaction was performed using TaqMan MicroRNA Assays (Applied Biosystems) or SYBR Premix Ex Taq (TAKARA) for miRNA and mRNA analyses, respectively. All values were normalised to human RNU6B or $\beta$-actin endogenous controls, respectively, and run in triplicate. The sequences of primers could be found in Supplementary Table 4. Amplification and detection were performed using a CFX96 Real-Time PCR System (Bio-Rad, Hercules, CA, USA).

Northern blot. MiRNA northern blot assays was performed using the MiRNA Northern Blot Assay Kit (Signosis, Sunnyvale, CA, USA) according to the manufacturer's protocol and as described in Yu et al (2007). Total RNAs were extracted as described above. High-sensitive miRNA probe used was follows: miR-200b: $5^{\prime}$-TCA TCATTACCAGGCAGTATTA-3'; miR-200c: 5'-TCCATCATTAC CCGGCAGTATTA-3'; RNU6B: 5'-AGTATATGTGCTGCCGAA GCGAGCAC- $3^{\prime}$. The image was acquired using a FluorChem FC2 imager (Alpha Innotech Corp, San Leandro, CA, USA). Expression of SUZ12/ROCK2 mRNA was assessed using DIG luminescent detection kit (Roche, Shanghai, China) as described (Almontashiri et al, 2013).

Wound healing assay. Cells $\left(1 \times 10^{6}\right.$ per well $)$ were cultured in 6 -well plates overnight, and then transfected with miR-200b/c mimics or antagomir and the respective controls. After $24 \mathrm{~h}$ of transfection, the cell layer was scratched with a sterile plastic tip, washed with PBS two times, and then cultured for $24 \mathrm{~h}$ with medium containing $1 \%$ FBS. Images of the plates were taken under a microscope.

Invasion assay. Transwell inserts (24-well insert, $8 \mu \mathrm{m}$ pore size; Corning Inc., Corning, NY, USA) were used to determine the invasion capacity in vitro. Inserts were precoated with extracellular matrix gel (BD Biosciences, Bedford, MA, USA). After transfection, cells were serum starved in serum-free medium overnight, and then resuspended in medium containing $0.1 \%$ bovine serum albumin and placed in the upper chambers in triplicate. Lower chambers were filled with $10 \%$ FBS as the attractant, and the cells were incubated for $48 \mathrm{~h}$. Then, the cells were removed from the upper surface of the membrane, and cells on the lower surface were fixed and stained with $0.4 \%$ paraformaldehyde and $0.1 \%$ crystal violet, respectively. Then, the stained cells were counted under a light microscope. 
Liver metastasis model of cholangiocarcinoma cells in BALB/c nude mice. Female $\mathrm{BALB} / \mathrm{c}$ nude mice, 4-6 weeks old, were purchased from the Animal Laboratory Unit of Peking Union Medical College (Beijing, China). The mice were maintained under standard conditions and reared according to the institutional guidelines for animal care. Lentivirus-transfected cells $\left(1 \times 10^{5}\right)$ were suspended in serum-free medium and then injected into the involucrum of the spleen. The mice underwent a lienectomy at 20 min after injection. The weight of the mice was measured once a week.

Xenograft experiments. A total of $1 \times 10^{5}$ transfected cells were subcutaneously injected into the right armpit of $\mathrm{BALB} / \mathrm{c}$ nude mice. The weight of the mice and the diameter of tumours were measured every week.

Drug resistance experiments. A total of $2 \times 10^{5}$ transfected cells were first seeded into one well of a 24 -well plate in the normal medium and cultured overnight. Then, the cells were treated with 5 -fluorouracil at the indicated concentrations. At 24, 48, and $72 \mathrm{~h}$, the survival rate was detected using a WST Cell Counting Kit- 8 (Dojindo, Kumamoto, Japan), and $\mathrm{IC}_{50}$ values were calculated following the manufacturer's instructions.

Sphere culture and differentiation. Cells (1000 cells per ml) were cultured in suspension in serum-free DMEM-F12 (Hyclone, Logan, UT, USA), supplemented with B27 (1:50; Invitrogen, Carlsbad, CA, USA), $20 \mathrm{ng} \mathrm{ml}^{-1}$ epidermal growth factor (PeproTech EC, London, UK), $100 \mathrm{ng} \mathrm{ml}^{-1}$ basic fibroblast growth factor (PeproTech EC), and $100 \mathrm{ng} \mathrm{ml}^{-1}$ leukaemia inhibitory factor (Chemicon, Billerica, MA, USA) as described elsewhere (Hermann et al, 2007). Differentiation experiments were carried out as described (Yu et al, 2007).

Flow cytometry. Transfected cells were dissociated, washed two times with BD PharMingen Stain Buffer (BD Bioscience), and resuspended. Antibodies were added to the cells followed by incubation for $30 \mathrm{~min}$ at $4{ }^{\circ} \mathrm{C}$. The antibodies used were $\mathrm{CD} 24-$ FITC, CD44-APC, and CD133-PE (BD Bioscience) each at $10 \mu \mathrm{l}$ per $1 \times 10^{6}$ cells. Flow cytometry was performed using a FACSAria (BD Immunocytometry Systems, Franklin Lakes, NJ, USA).

Luciferase reporter assay. The $3^{\prime}$-UTRs of eight putative SUZ12/ ROCK2 genes were selected according to the genomic fragment of TargetScan containing the predicted miR-200b/c/429 binding sites. The mutant SUZ12/ROCK2 3'-UTR construct was designed to mutate three intermittent nucleotides complementary to the miR$200 \mathrm{~b} / \mathrm{c} / 429$ seed region. The TFK-1 cells were cultured in 24-well plates and transfected with $400 \mathrm{ng}$ of either wild-type and one mutant pMIR/SUZ12 or three mutant pMIR/ROCK2 plasmids carrying firefly luciferase, together with $100 \mathrm{ng}$ pRL-TK vector (Promega, Madison, WI, USA) carrying renilla luciferase. Transfection was performed using Lipofectamine 2000 (Invitrogen). Relative luciferase activity was calculated at $48 \mathrm{~h}$ post-transfection by a Dual Luciferase Reporter Assay (Promega).

Statistical analysis. Data are presented as the mean \pm standard error. Statistically significant differences were determined by the Student's $t$-test. Statistical analyses were performed by SPSS Software (version 19.0) (SPSS Inc, Chicago, IL, USA). A twotailed value of $P<0.05$ was considered to indicate a statistically significant result.

\section{RESULTS}

Differential expression of miRNAs in cholangiocarcinoma patients, and validation of microarray results by RT-qPCR. To identify miRNAs that are differentially expressed in cholangiocarcinoma, we compared the expression of miRNAs in three cholangiocarcinoma samples and the corresponding normal bile duct tissues using an Agilent miRNA Microarray. We found that 15 miRNAs were overexpressed and six miRNAs were underexpressed in the cholangiocarcinoma tumour group compared with that in the controls (Figure 1A). The 15 overexpressed miRNAs in the cholangiocarcinoma group were 2.25 - to 42222 -fold overexpressed compared with the control group, and the six underexpressed miRNAs were 6.39- to 4032fold underexpressed compared with the controls (Supplementary Table 2). Next, we predicted the possible targets of this differential expression and identified 2844 target genes. These target genes were then analysed for biological process and molecular function using information available in the Gene Ontology (GO) database. As shown in Supplementary Figure $1 \mathrm{~B}$ and Supplementary Table 5, there were 78 upregulated GO terms and 82 downregulated terms. To determine whether the abnormal expression of these genes was due to dysregulated signalling pathways, we performed pathway analyses and found 20 upregulated pathways and 30 downregulated pathways in relation to the dysregulated genes (Supplementary Figure 1C and Supplementary Table 6). Using the significant pathway terms, a network of pathways and genes was generated using the KEGG database (Supplementary Figure 2A and B). Lastly, we identified the crucial target genes regulated by the differentially expressed miRNAs, including miR-200b and miR-200c (Supplementary Figure 1A). Three miRNAs, miR-200b, miR-200c, and miR-429, were selected for validation of the microarray results by RT-qPCR and northern blotting. The data analysis showed that miR-200b, miR-200c, and miR-429 were underexpressed in the cholangiocarcinoma group compared with the controls (Figure $1 \mathrm{~B}$ and Supplementary Figure 7A). The data showed that the reduced expression of miR-200b and miR-200c was significant (Figure $1 \mathrm{C}, P=0.038$ and $P=0.019$, respectively), whereas that of miR-429 was not (Figure $1 \mathrm{C}, P=0.057$ ). The RT-PCR and northern results were consistent with those of the microarray analysis. Therefore, miR-200b and miR-200c were selected for further study.

Ectopic miR-200b/c inhibits the migration and invasion of cholangiocarcinoma cells. We determined whether miR-200b/c can alter the migration and invasion capacities of cholangiocarcinoma cells. As expected, the transfection of miR-200b/c mimics and antagomir into TFK-1 cells resulted in a substantial increase (by mimics) and decrease (by antagomir) of miR-200b/c expression, respectively, compared with that in the NC-transfected cells (Figure 2A, $P<0.001)$. An invasion assay showed that the overexpression of $\mathrm{miR}-200 \mathrm{~b} / \mathrm{c}$ resulted in a reduction in the invasion rate of TFK- 1 cells compared with that of the control (Figures 2C and B, $5.04 \pm 0.11$ - and $3.12 \pm 0.03$-fold, respectively, $P<0.001)$. The TFK-1 cells transfected with the miR-200b/c mimics also slowly closed wound gaps compared with the control (Figure 2D and Supplementary Figure 4, 2.13 \pm 0.24 - and $1.92 \pm 0.03$-fold, respectively, $P<0.001)$. Similar results were observed using QBC939 cells (Figure 2E and Supplementary Figure $3 \mathrm{~A}$ and $\mathrm{B}$ ). In contrast, the knockdown of miR-200b/c promoted the invasion of the cholangiocarcinoma cells (Figure 2C): miR-200b/c knockdown in TFK-1 cells resulted in significant gap closure in the wound healing assay (Figure 2D, $0.69 \pm 0.01$ - and $0.65 \pm 0.03$-fold, respectively, $P<0.001)$ and a increase in cell invasion compared with that of the antagomir-NC group (Figure 2B, $1.96 \pm 0.10$ - and $3.07 \pm 0.19$-fold, respectively, $P<0.001)$. Moreover, we confirmed an effect of miR-200b/c on cholangiocarcinoma's metastatic capacity in vivo. We transfected TFK-1 cells with a lentivirus-based expression vector for either the overexpression or downregulation $\mathrm{miR}-200 \mathrm{~b} / \mathrm{c}$, and then established a liver metastasis model of cholangiocarcinoma using these 
A

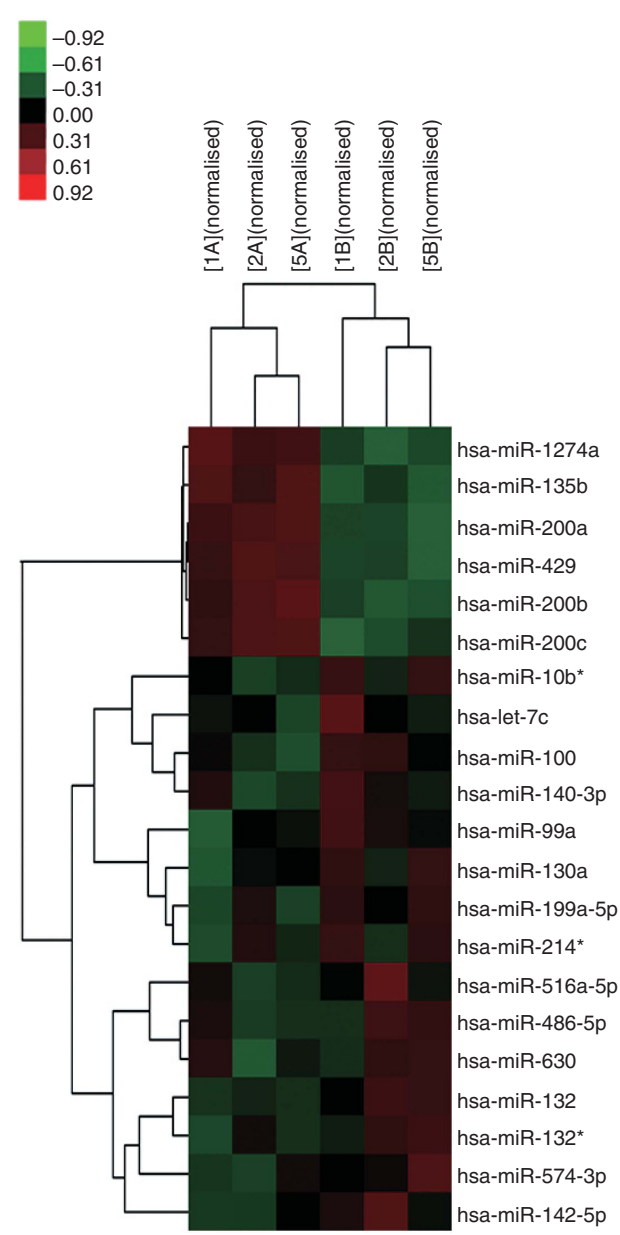

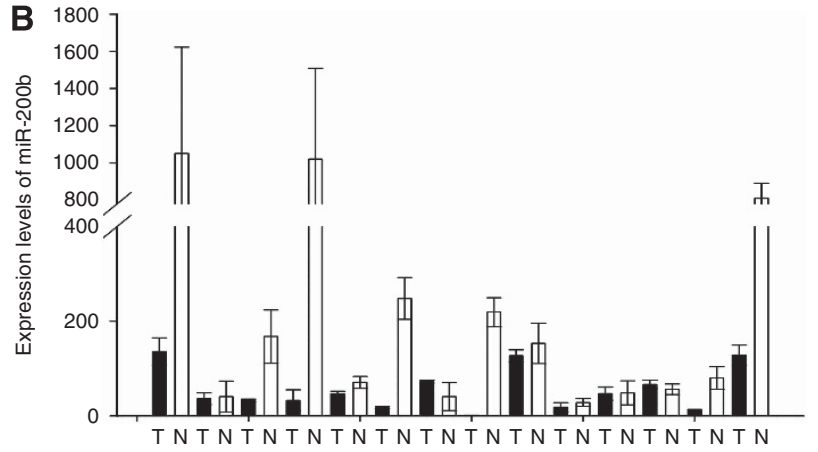
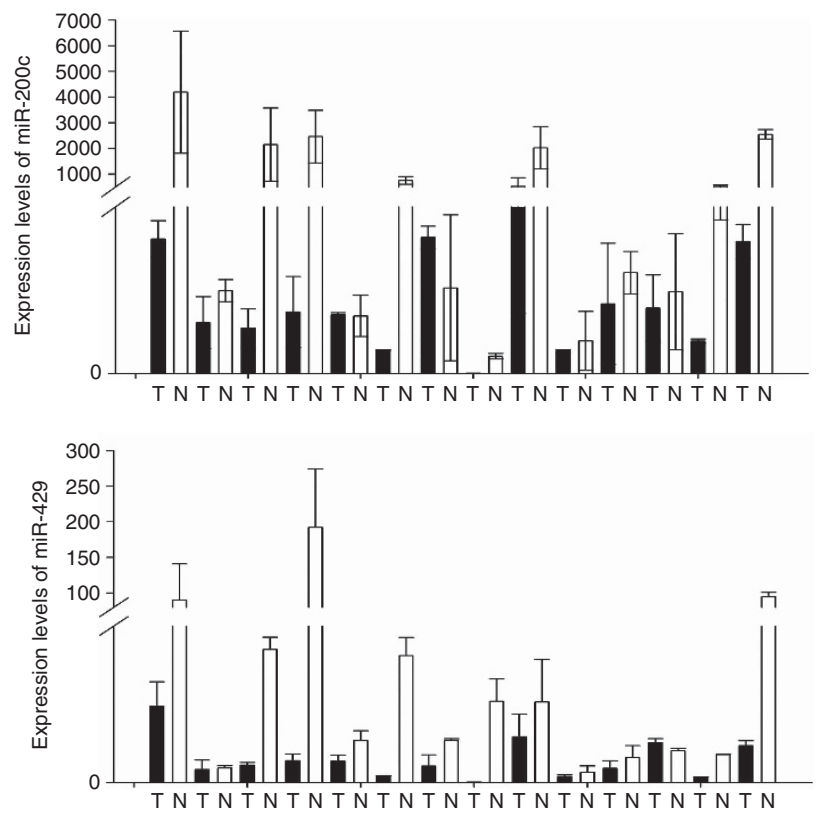
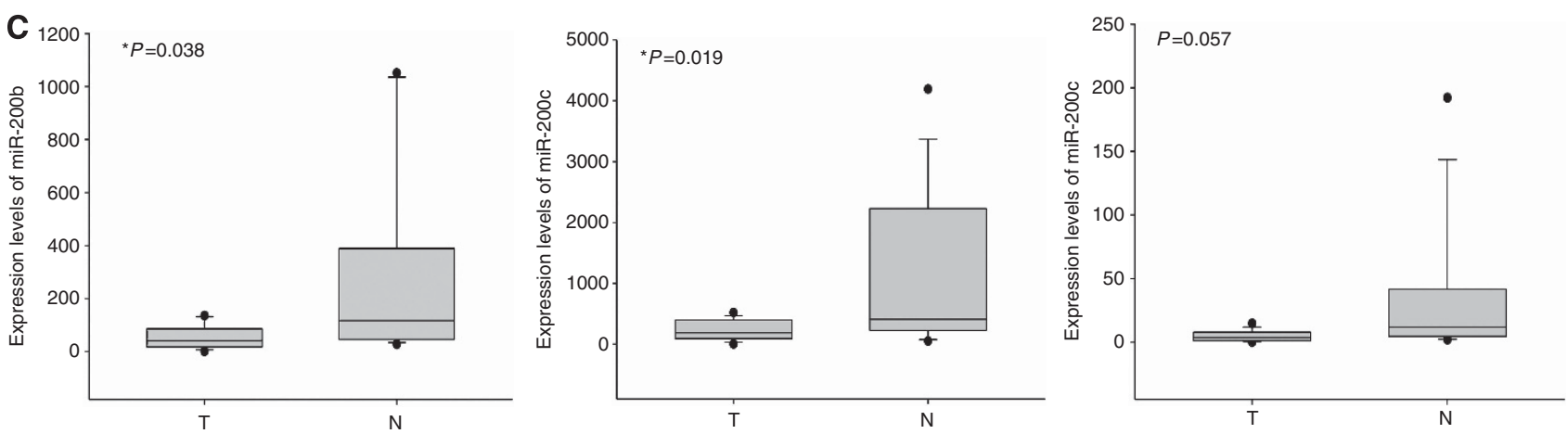

Figure 1. Differential expression of miRNAs in three cholangiocarcinoma samples and matching normal bile duct tissues as determined by an Agilent miRNA microarray. (A) Heatmap of miRNA profiling. A line indicates a microRNA gene, and a column indicates a sample. Red reflects a high expression value, whereas green reflects a low expression value. (B) Validation of differential miR-200b/c/429 expression in 14 cholangiocarcinoma and matching normal bile duct tissues by RT-qPCR. The expression levels of miR-200b, miR-200c, and miR-429 are shown.

(C) Collected data from 14 pairs of cholangiocarcinoma patient tissues show the expression of miR-200b/c/429 compared with that in their normal bile duct tissue ${ }^{*} P<0.05$. Abbreviations: $N=$ Normal bile duct tissue; $T=$ cholangiocarcinoma (tumour group).

transfected cells. The results showed that the upregulation of miR200b/c inhibited the distant metastasis of TFK-1 cells, whereas the knockdown of $\mathrm{miR}-200 \mathrm{~b} / \mathrm{c}$ promoted this metastatic process (Figure 3A). Because of the low resolution of MRI, we could not scan all the liver tumour metastases. Thus, we prepared serial sections of whole liver for haematoxylin and eosin (HE) staining, and observed the sections under a light microscope. According to the results, more micrometastases were detected in the miR-200b/ c-knockdown group, and miR-200b/c upregulation reduced the formation of micrometastases compared with the NC groups
(Figure 3A). Interestingly, miR-200c might have more of an influence on distant metastasis than miR-200b. In conclusion, these data show that $\mathrm{miR}-200 \mathrm{~b} / \mathrm{c}$ is an important participant in the regulation of the migration and invasion potentials of cholangiocarcinoma cells.

MiR-200b/c expression is associated with 5-fluorouracil chemoresistance in cholangiocarcinoma cells. To determine whether miR-200b/c expression is also associated with chemoresistance in cholangiocarcinoma, we measured the 

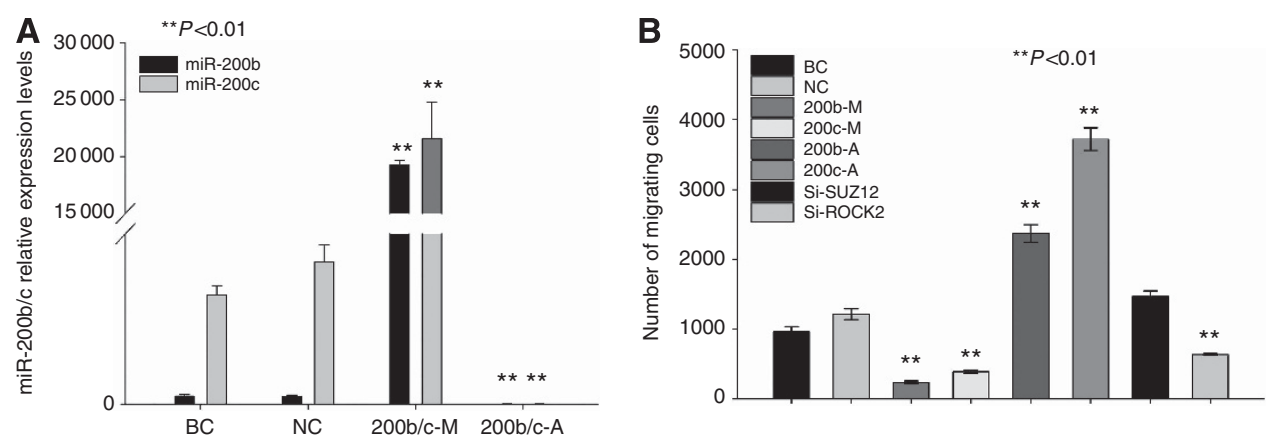

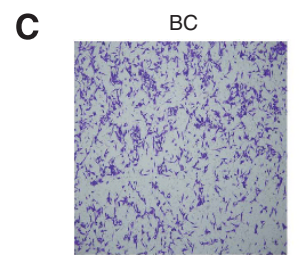

200b-M

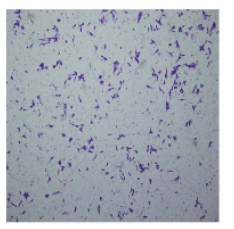

200b-A

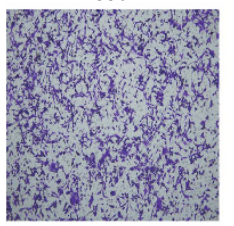

Si-ROCK2

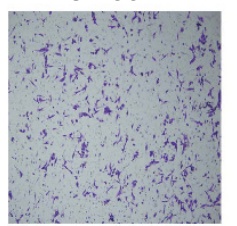

NC

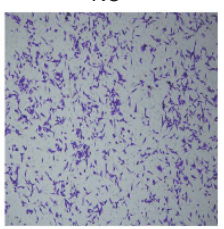

200c-M

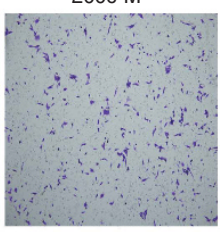

200c-A

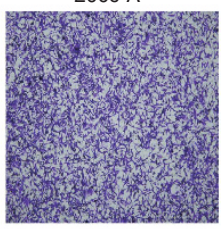

Si-SUZ12

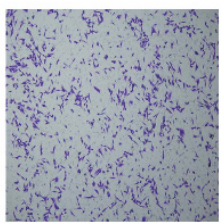

D
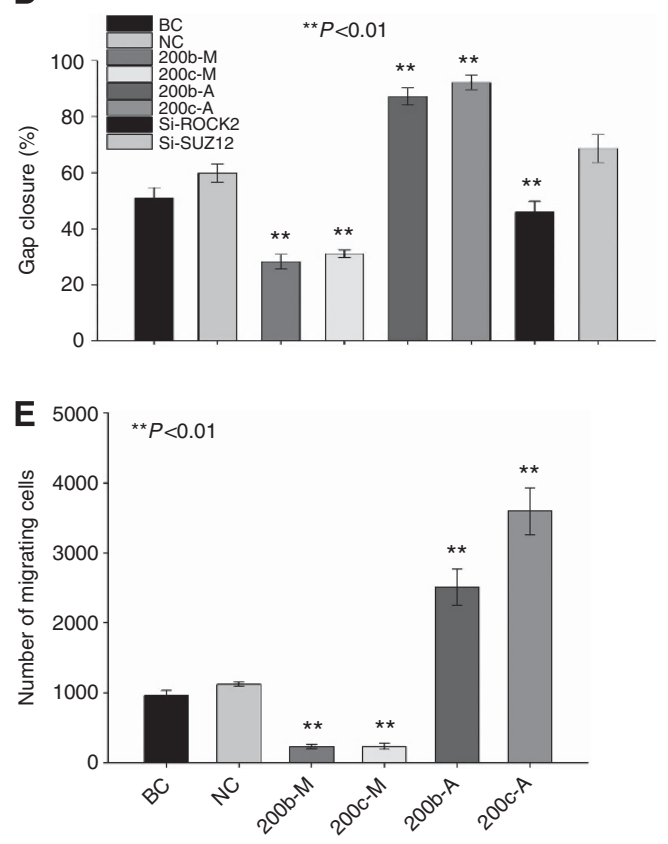

Figure 2. Ectopic miR-200b/c expression inhibits the invasion and migration of cholangiocarcinoma cells. The effects of miR-200b/c mimics and an antagomir are shown in (A). (C) Upregulated miR-200b/c expression inhibited the invasion capacity of TFK-1 cells, whereas downregulated miR$200 \mathrm{~b} / \mathrm{c}$ expression promoted the capacity. The number of invasive cells is shown in (B). Wound-healing assay of transfected TFK-1 cells: the proportion of gap closure in the wound healing assay is shown in (D). All the data were compared with those from the NC group. MiR-200b/c upregulation inhibited the invasion capacity of QBC939 cells, whereas downregulated miR-200b/c promoted this capacity. The number of invasive cells is shown in (E). Abbreviations: BC= blank control group; $N C=$ negative control group; 200b/c-M =200b/c-mimics group; 200b/c-A = 200b/ c-antagomir group.

expression of miR-200b/c in TFK-1 cells pretreated with 5 -fluorouracil, which is widely used for cholangiocarcinoma treatment. The expression level of $\mathrm{miR}-200 \mathrm{~b} / \mathrm{c}$ in the 5-fluorouracil-pretreated TFK-1 cells was lower than that in the untreated controls (Supplementary Figure 3C). We then transfected TFK-1 cells as described above and found that pretreatment with $\mathrm{miR}-200 \mathrm{~b} / \mathrm{c}$ mimics promoted the cytotoxic effects of 5-fluorouracil on TFK-1 cells compared with the 5 -fluorouracil $+\mathrm{NC}$ (Figure 4A). The $\mathrm{IC}_{50}$ values for the pre-miR-200b mimics +5 -fluorouracil and pre-miR-200c mimics +5 -fluorouracil groups at various time points are shown in Supplementary Table 3, with the $\mathrm{IC}_{50}$ value for the miR-200b/ c mimics +5 -fluorouracil group being significantly lower than that of the NC group. Conversely, the knockdown of miR-200b/ c by an antagomir aggravated the chemoresistance against 5-fluorouracil. As shown in Supplementary Table 3, the $\mathrm{IC}_{50}$ values for the pre-miR-200b antagomir +5 -fluorouracil and
pre-miR-200c antagomir +5 -fluorouracil groups at various time points were significantly higher than those of the $\mathrm{NC}+5$ fluorouracil. These data illustrate that $\mathrm{miR}-200 \mathrm{~b} / \mathrm{c}$ significantly enhances the cytotoxic effect of 5-fluorouracil on cholangiocarcinoma cells.

Ectopic miR-200b/c expression in cholangiocarcinoma cells influences their self-renewal in vitro and tumorigenic potential in vivo, and CD133 expression can be influenced by exogenous $\mathrm{miR}-200 \mathrm{~b} / \mathrm{c}$ expression. The relationship between miR-200b/c and metastasis/chemoresistance had been demonstrated, and it is well-known that there are multiple steps in tumourigenesis. To demonstrate whether miR-200b/c expression affects other aspects of cholangiocarcinoma tumorigenicity in vivo and in vitro, we transfected TFK-1 cells with the lentivirus-based expression vector, as described above. To investigate the effects of miR-200b/c on tumour formation, we next subcutaneously injected TFK-1 cells 
A
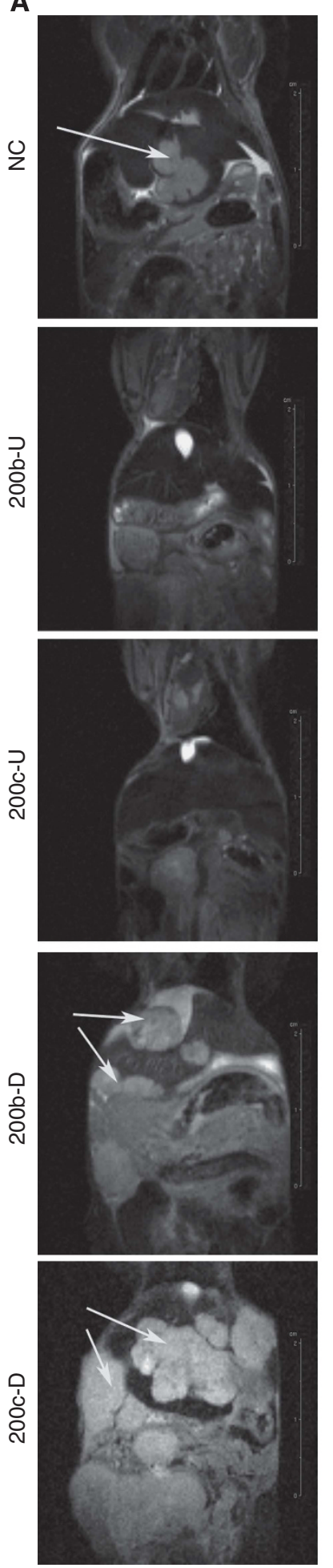
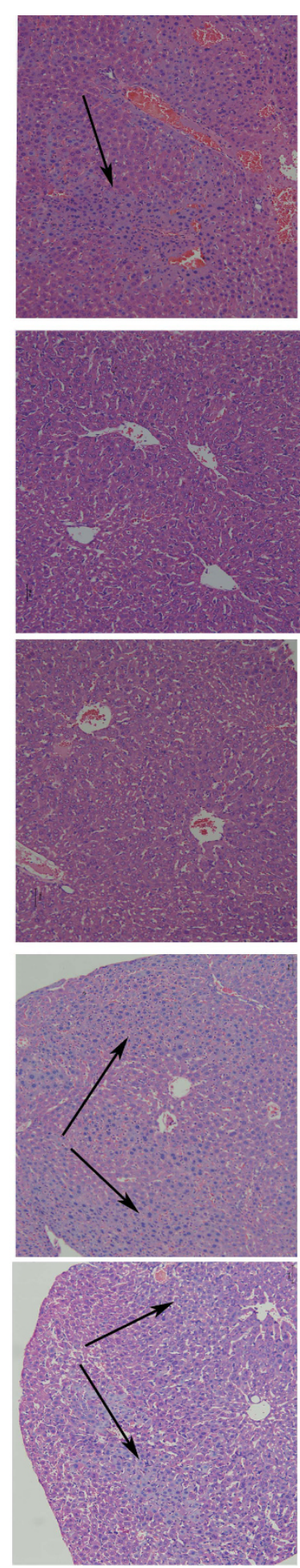

B

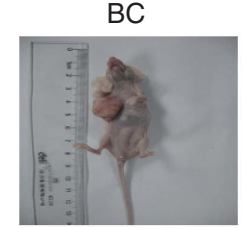

$200 b-U$

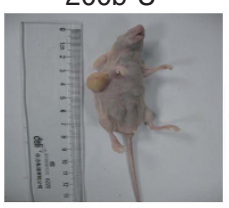

200b-D
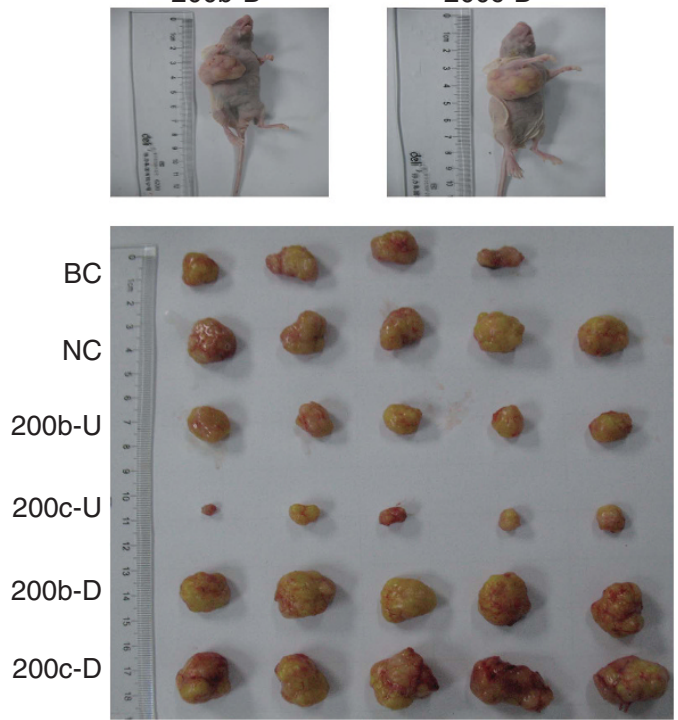

C
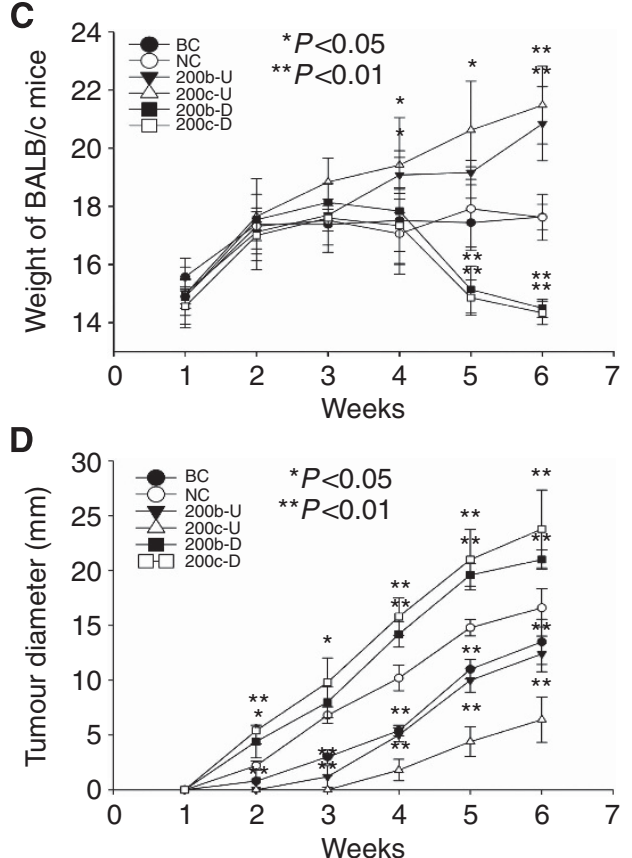

Figure 3. Ectopic miR-200b/c expression inhibits the migration and distant metastasis capacities of TFK-1 cells. (A) Lentivirus-transfected TFK-1 cells were used to construct a liver metastasis model of cholangiocarcinoma cancer cells. Images of the MRI scans are shown on the left line. Whole-liver serial sectioning for HE staining of the liver metastasis BALB/C-nude mice model. The micrometastases are shown on the right line. (B) Different tumour-initiating capacity of transfected TFK-1 cells. All the BALB/c-nude mice were killed at 6 weeks after injection. The weight data are shown in (C). The tumour diameters are shown in (D). All of the data were compared with those from the NC group. Abbreviations: $B C=b$ blank control group; $N C=$ negative control group; 200b/c-U =200b/c-upregulated lentivirus group; $200 \mathrm{~b} / \mathrm{c}-\mathrm{D}=200 \mathrm{~b} / \mathrm{c}$ downregulated lentivirus group. 

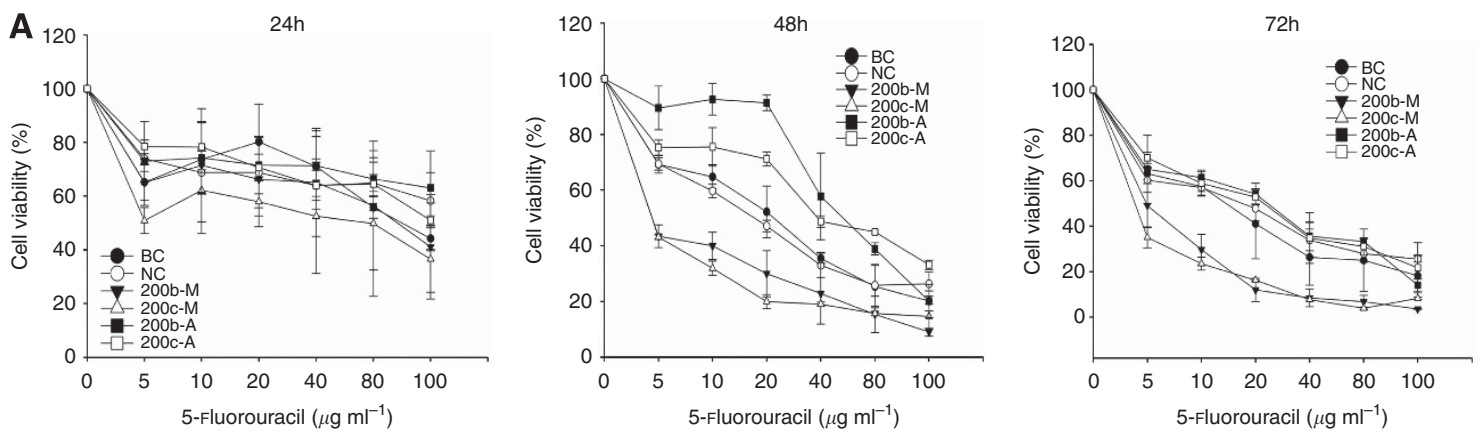

B
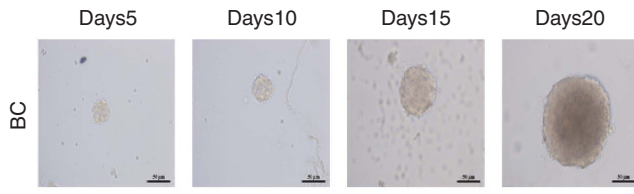

C 600
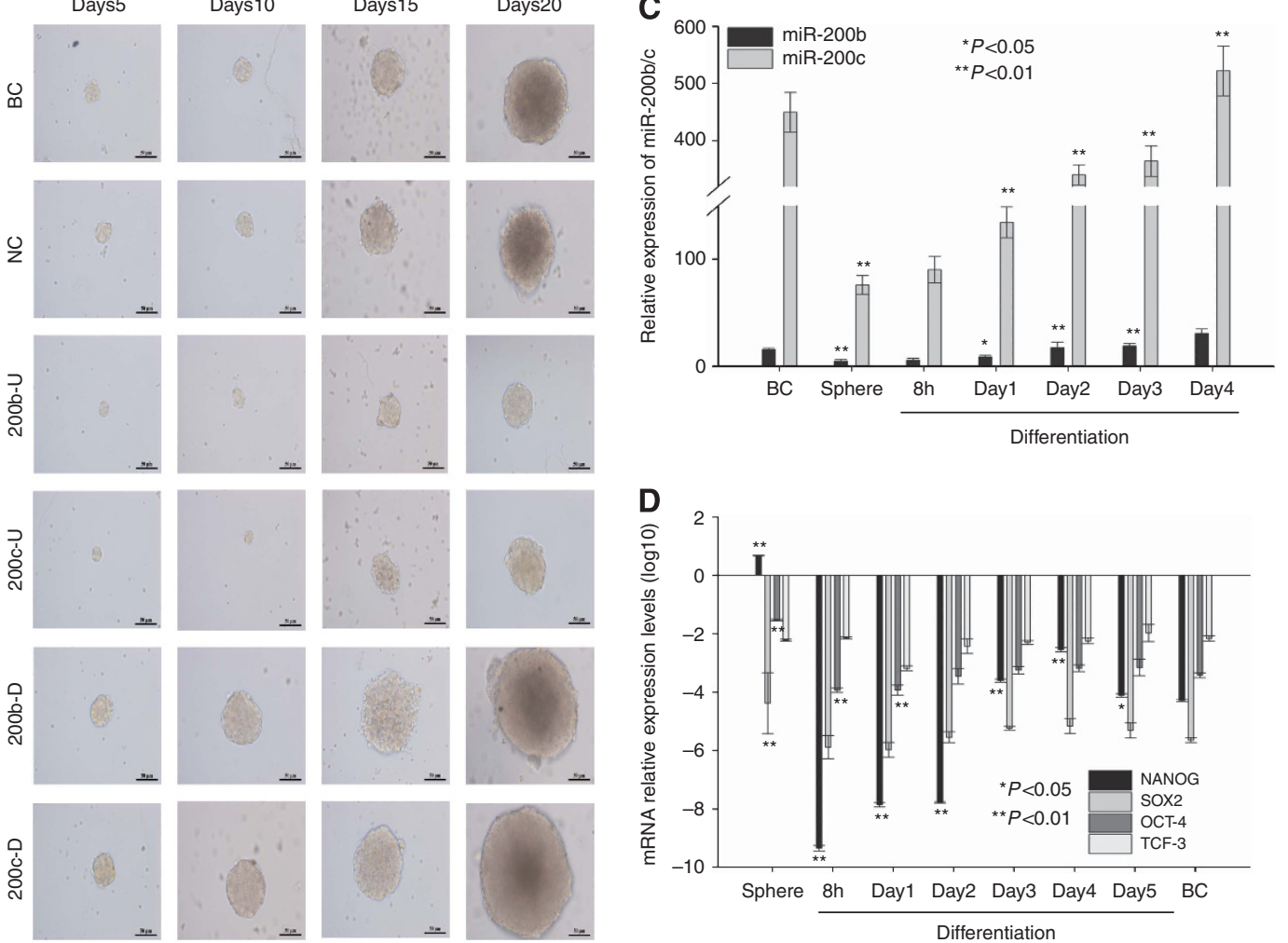

E
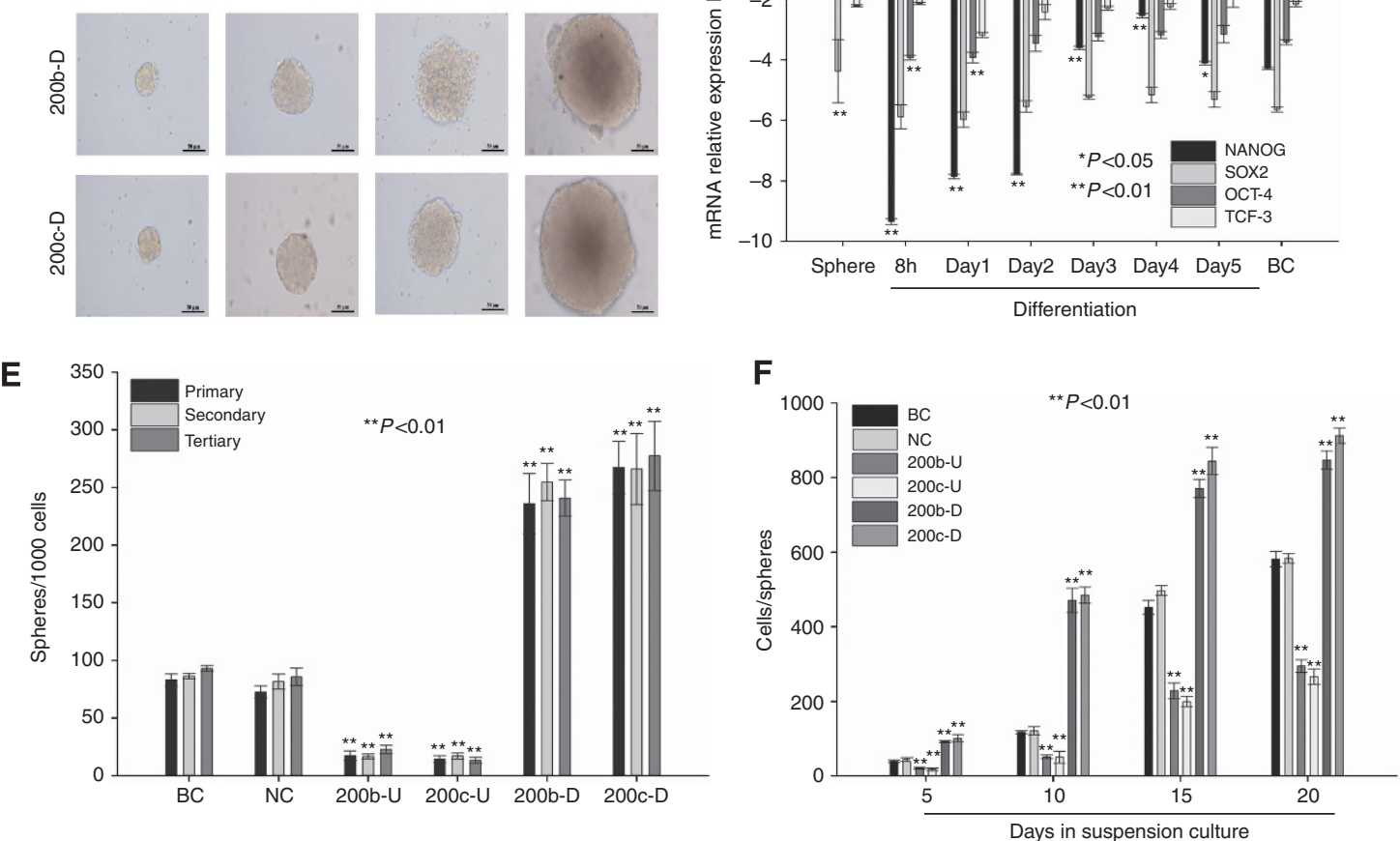

Figure 4. (A) 5-Fluorouracil chemoresistance. The proportion of cell viability is shown at 24, 48, and $72 \mathrm{~h}$. (B) Different transfected TFK-1 cells were used for sphere formation and are shown at various time points (5, 10, 15, and 20 days). The expression of miR-200b and miR-200c (C) was detected during sphere formation and differentiation culture. The expression of NANOG, SOX2, TCF3, and OCT-4 mRNA during sphere formation and differentiation culture is shown in (D). The cell per sphere values (F) indicate the dimensions of the spheres. Sphere per 1000 cells (E) shows the capacity for sphere formation. All the data were compared with the data from the NC group.

transfected with the control, $\mathrm{miR}-200 \mathrm{~b} / \mathrm{c}$ overexpression, and miR$200 \mathrm{~b} / \mathrm{c}$ knockdown vectors into the right armpit of BALB/c nude mice. Strikingly, even after 3 weeks, injection of $1 \times 10^{5} \mathrm{miR}-200 \mathrm{~b} /$ c-knockdown cells resulted in large tumours, whereas the miR- 200b/c-overexpressing cells formed smaller tumours in five out of five mice compared with the control groups (Figure 3B, $P<0.001$ ). The tumour size was also dramatically altered, as shown in Figure 3D, the miR-200b/c-overexpressing cells formed tumours at 
a much slower rate than the NC group. The miR-200b/cknockdown cells showed the opposite trend $(P<0.001)$. The weight of the mice was also statistically significance from week 4 (Figure 3C, $P<0.01$ ). Figure $3 \mathrm{~B}$ shows the different tumourinitiating capacities of the transfected cholangiocarcinoma cells. All these results confirm that the tumour-initiating capacity of TFK-1 cells is associated with low miR-200b/c expression, in support of our future research objectives.

To investigate whether miR-200b/c is important for influencing the tumorigenic properties of cholangiocarcinoma cells, we transfected TFK-1 cells with the lentiviral vectors described above and cultured the transfected cells in suspension under serum-free conditions to generate spheres. The number of spheres reflects the self-renewal capacity of the cells in vitro, whereas the number of cells per spheres indicates the self-renewal capacity of each sphere-generating cell ( $\mathrm{Yu}$ et al, 2007). Over three passages, the $\mathrm{miR}-200 \mathrm{~b} / \mathrm{c}$-overexpressing cells formed fewer spheres than the cells in the NC group (Figure 4E, 17.33 $\pm 4.04,16.67 \pm 2.08$, and $22.67 \pm 3.51$ for each passage of miR-200b-overexpressing cells, $14.33 \pm 3.05,17.0 \pm 2.65$, and $13.33 \pm 2.52$ for each passage of miR-200c-overexpressing cells, and $72.67 \pm 5.13,81.67 \pm 6.5$, and $85.67 \pm 7.5$ for each passage of NC cells, $P<0.001)$. In contrast, the miR-200b/c-knockdown cells formed more spheres than the NC group over three passages (Figure 4E, 236 $\pm 26,254.7 \pm 16$, and $240 \pm 15.6$ for each passage of miR-200b-knockdown cells, and $267 \pm 22.7,266 \pm 30$, and $277.3 \pm 29.96$ for each passage of miR-200c-knockdown cells, $P<0.001)$. As shown in Figures $4 \mathrm{~B}$ and $\mathrm{F}$, after 20 days of culture, the miR-200b/c-knockdown cells formed larger spheres than the controls cells $(847 \pm 24.6$ and $912.67 \pm 20.31$ vs $583.67 \pm 12.4$, respectively, $P<0.001$ ), whereas the miR-200b/c-overexpressing cells formed smaller spheres than the controls cells $(294.67 \pm 17.04$ and $266.33 \pm 20.6$, respectively, $P<0.001)$. To imitate the differentiation of cancer cells, the dissociated spheres were plated on collagen IV in serum under differentiating conditions. After 6 days under differentiating conditions, the expression levels of differentiation markers OCT-4, NANOG, and SOX2 were decreased (Figure 4D), whereas the levels of $\mathrm{miR}-200 \mathrm{~b} / \mathrm{c}$ decreased after sphere formation and increased during differentiation (Figure 4C). Taken together with the slower growth rate and size of the $\mathrm{miR}-200 \mathrm{~b} / \mathrm{c}$-overexpressing spheres and the inability to passage the cells as spheres, these data suggest that miR-200b/c-overexpressing spheres may have undergone the first step toward losing their self-renewal capacity. Conversely, the miR-200b/c-knockdown spheres appeared to have a greater capacity for sphere formation, indicating a higher self-renewal capacity.

Self-renewal capacity is an important characteristic of tumourigenesis and is also a potential stem-like property of cancer cells. Our previous studies showed that CD24, CD44, and CD133 are probable stem cell surface markers of cholangiocarcinoma (Wang et al, 2011a, b). As our above results confirmed that $\mathrm{miR}-200 \mathrm{~b} / \mathrm{c}$ expression might be associated with the self-renewal capacity of cholangiocarcinoma, $\mathrm{miR}-200 \mathrm{~b} / \mathrm{c}$ may have a potential effect on stem-like cholangiocarcinoma cells. Therefore, we validated these results using flow cytometry by incubating the transfected TFK-1 cells, as described above, with antibodies against CD24, CD44, and CD133. As shown in Figures 5A and B, the proportion of $\mathrm{CD} 133+$ cells among the miR-200b/coverexpressing cells was lower than that in the $\mathrm{NC}$ $(29.72 \pm 0.15 \%, 16.55 \pm 0.11 \%$ vs $72.77 \pm 1.89 \%)$. However, the proportion of CD133 + cells among the miR-200b/c-knockdown cells was high $(89.47 \pm 1.81$ and $87.66 \pm 1.19 \%)$. Furthermore, the proportion of CD24 + and CD44 + cells was not related to miR200b/c expression (Supplementary Figure 6B). Collectively, these data show that CD133 is a potential CSC marker of cholangiocarcinoma (Wang et al, 2011a), which can be inhibited by miR$200 \mathrm{~b} / \mathrm{c}$.
SUZ12 and ROCK2 are direct targets of $\mathrm{miR}-200 \mathrm{~b} / \mathrm{c}$. According to our results, miR-200b/c affects migration, invasion, chemoresistance, tumour growth, and self-renewal capacities. Previous studies have shown that SUZ12 is a crucial CSC gene and a direct target of miR-200b in breast cancer cells. ROCK2 is an important downstream gene of the Rho/ROCK2 pathway, participating in the regulation of cytoskeletal signalling events and is crucial for cell motility (Supplementary Figures 2A and B). A TargetScan analysis (TargetScan Human 6.0) revealed that SUZ12 contains a classical and evolutionarily conserved $\mathrm{miR}-200 \mathrm{~b} / \mathrm{c}$ binding site in its $3^{\prime}$-UTR; ROCK2 contains one conserved and two poorly conserved miR-200b/c binding sites (Supplementary Figure 5). The ectopic expression of miR-200b/c in TFK-1 cells resulted in a reduction in SUZ12 and ROCK2 expression, whereas miR-200b/c inhibition led to increased expression (Figure 5C). However, their mRNA expression levels were unaffected by transfection (Supplementary Figure 7D). To further confirm whether SUZ12 and ROCK2 are direct targets of $\mathrm{miR}-200 \mathrm{~b} / \mathrm{c}$, we detected the expression of the SUZ12 and ROCK2 proteins in 12 cholangiocarcinoma samples and the corresponding normal bile duct tissues using western blotting and immunohistochemistry. The results showed that both SUZ12 and ROCK2 were overexpressed in the cholangiocarcinoma tissues: 8 of 12 and 9 of 12 tumour tissues showed increased SUZ12 and ROCK2 levels, respectively, compared with the matched normal bile duct tissues (Figure 5D). Immunohistochemistry also showed that the protein expression levels of SUZ12 and ROCK2 were higher in the tumour tissues than in the normal bile duct tissues (Figure 5E, Supplementary Figures $3 \mathrm{D}$ and $\mathrm{E}, P=0.018$ and $<0.001$, respectively). Regardless, there was little significant difference in the mRNA expression levels of SUZ12/ROCK2 between the cholangiocarcinoma and normal bile duct groups (Supplementary Figure 7B), suggesting that $\mathrm{miR}-200 \mathrm{~b} / \mathrm{c}$ might silence these two target only in a post-transcriptional manner. To further confirm our results, the $\mathrm{miR}-200 \mathrm{~b} / \mathrm{c}$ binding sites of SUZ12 and ROCK2 were each cloned into a luciferase reporter. When the SUZ12 wild-type sequence, but not a mutant sequence, was transfected into TFK-1 cells, we found that the ectopic expression of miR-200c resulted in a decrease in luciferase activity (SUZ12 has been reported to be direct target of miR-200b; Iliopoulos et al, 2010). Similar data were observed for ROCK2, showing that the forced expression of $\mathrm{miR}-200 \mathrm{~b} / \mathrm{c}$ in TFK-1 cells results in a decrease in luciferase activity using conserved wild-type binding sites compared with a mutant sequence (Figures $6 \mathrm{~A}$ and $\mathrm{B}$ ) and also indicating that the conserved predicted binding sites of $\mathrm{miR}-200 \mathrm{~b} / \mathrm{c}$ in the $3^{\prime}-\mathrm{UTR}$ of ROCK2 were functional. In conclusion, the above data demonstrate that SUZ12 and ROCK2 are direct target genes of $\mathrm{miR}-200 \mathrm{~b} / \mathrm{c}$.

SUZ12 and ROCK2 are potentially involved in the miR-200b/cregulated properties of cholangiocarcinoma. Previous studies have concluded that miR-200-Zeb1/2-E-cadherin is correlated with the migration and invasion capacities of cancer cells. To determine whether SUZ12 and ROCK2 are related to these capacities, we transfected TFK-1 cells with SUZ12 and ROCK2 siRNAs to knockdown their expression and then detected the invasion capacity using a Transwell assay. The knockdown of SUZ12 did not affect the invasion capacity of TFK-1 cells: the number of invasive cells had no statistical significance compared to that of the control. In contrast, ROCK2 knockdown reduced the number of invasive cells compared with the controls (Figures $2 \mathrm{~B}$ and $\mathrm{C}$ ). Furthermore, we transfected TFK-1 cells with ROCK2 and $Z E B 1 / 2$ siRNAs (Figure $6 \mathrm{C}$ ), to confirm their involvement in the migration and invasion capacities of cancer cells. However, the silencing of neither ROCK2 nor ZEB1/2 in the miR-200b/cknockdown cells could recover the invasive capacity compared with that of the controls. In addition, the silencing of both 
A
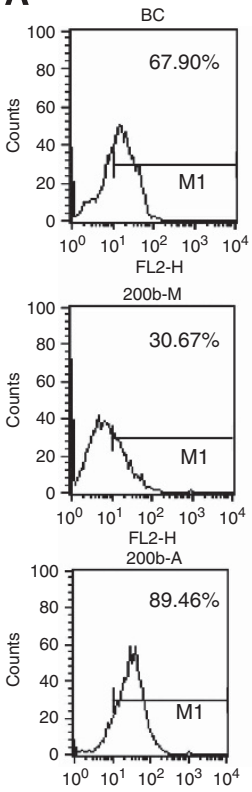
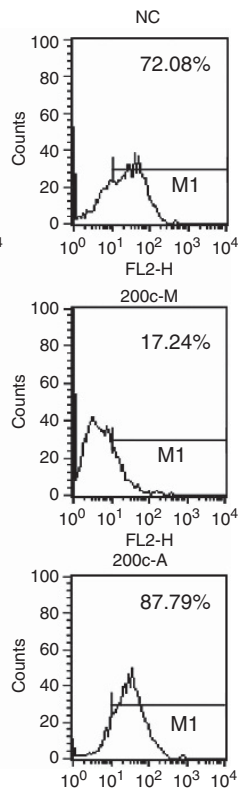

B

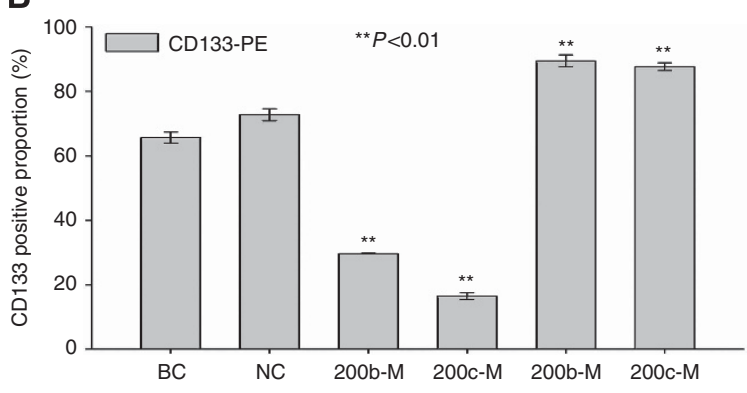

C
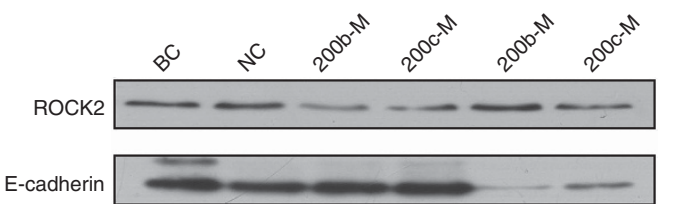

SUZ12

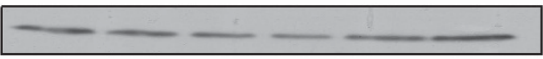

$\beta$-Actin

D
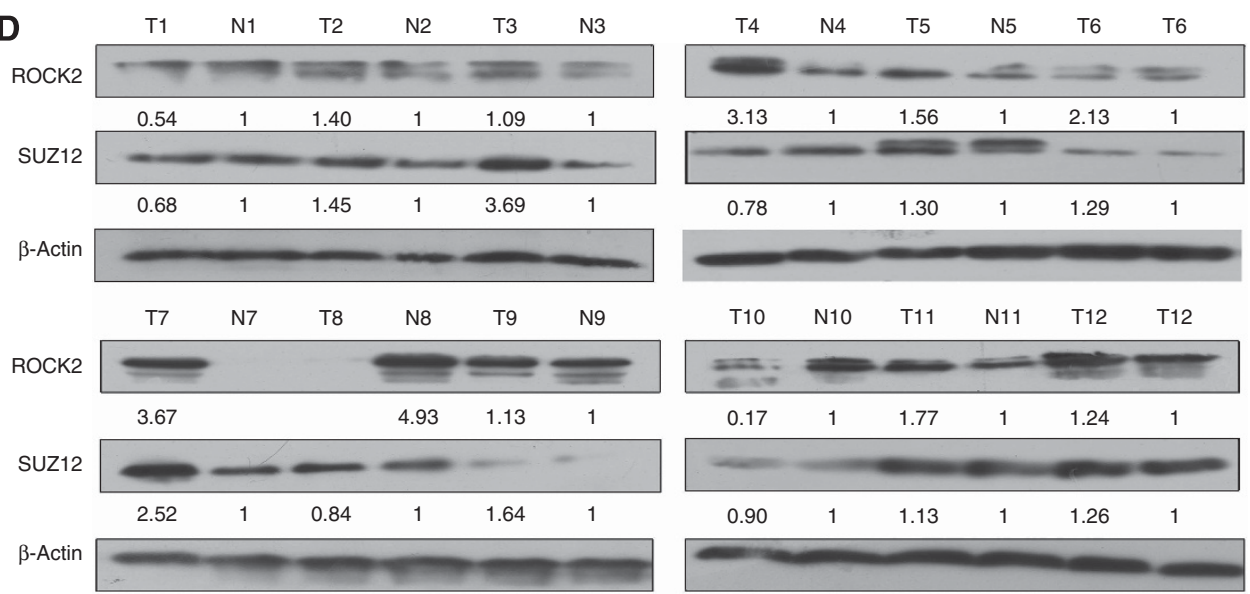

E T

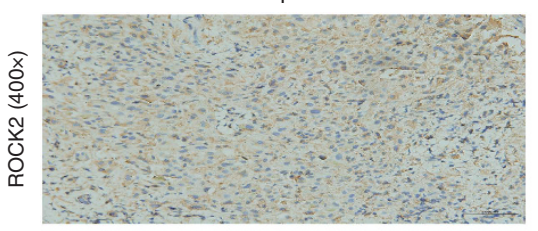

N
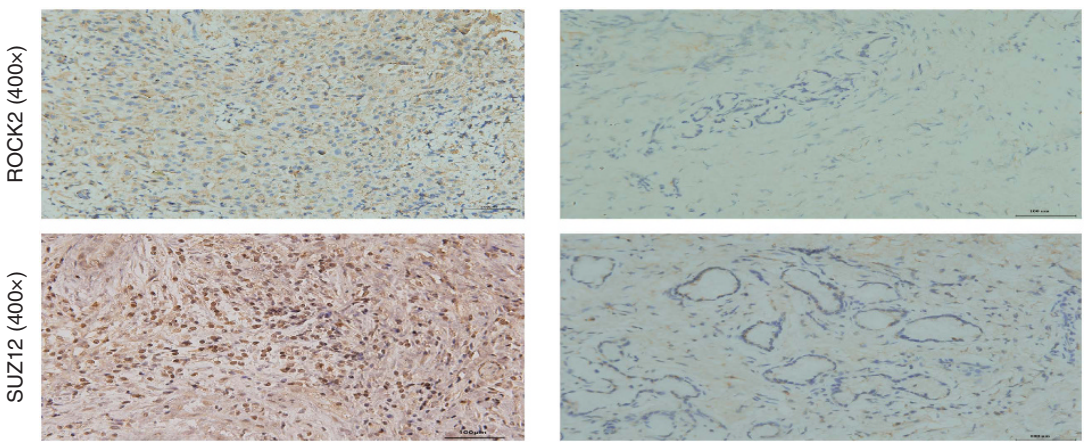

Figure 5. (A) Proportion of CD133 + cells among transfected TFK-1 cells. At $48 \mathrm{~h}$ after transfection with miR-200b/c mimics/antagomir, the TFK-1 cells were resuspended, and the expression of CD133 was detected on the cell membrane. All the proportions are relative to the blank control and are shown in (B). (C) Protein expression of SUZ12, ROCK2, and E-cadherin. $\beta$-Actin was used as the control. All the data were compared with the data from the NC group. (D) Protein expression of SUZ12 and ROCK2 in 12 cholangiocarcinoma and matching normal bile duct tissues. (E) Immunohistochemistry of normal bile duct and cholangiocarcinoma tissues. The left and right images are the cholangiocarcinoma and normal bile duct tissues, respectively.

ROCK2 and ZEB1/2 resulted in the lowest number of invasive cells (Supplementary Figures 6A and D). Collectively, the results showed that the effect of $\mathrm{miR}-200 \mathrm{~b} / \mathrm{c}$ on the invasion capacities of cholangiocarcinoma cells is mediated by both the Zeb1/2-Ecadherin axis and also the ROCK2 pathway, whereas SUZ12 is not associated with the migration and invasion capacities of cholangiocarcinoma cells. Furthermore, we transfected TFK-1 cells with $\mathrm{miR}-200 \mathrm{~b} / \mathrm{c}$ mimics/antagomir and detected selected downstream genes of the ROCK2 pathway. As shown in Figures $5 \mathrm{C}$ and $6 \mathrm{G}$, the overexpression of $\mathrm{miR}-200 \mathrm{~b} / \mathrm{c}$ decreased the protein expression level of ROCK2 and then increased the expression level of LIMK2. Increased LIMK2 could phosphorylate 

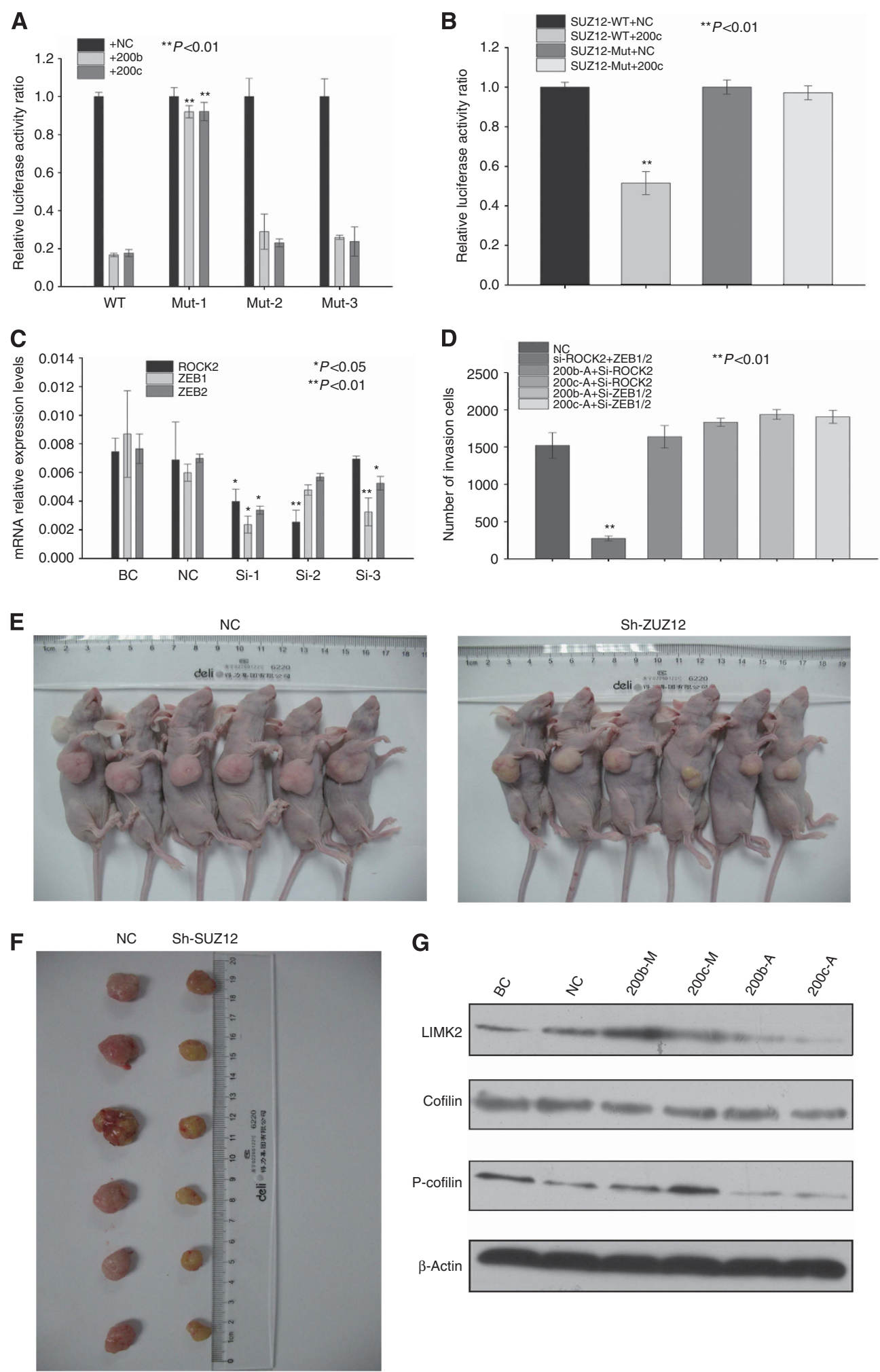

Figure 6. Luciferase reporter assays of SUZ12 and ROCK2 expression are shown in (A) and (B). The mRNA expression of ROCK2 and ZEB1/2 after transfection of siRNA is shown in (C). Effect on the invasion capacity of TFK-1 cells after transfection with si-ROCK2+miR-200b/c antagomir, si-ZEB1/2 + miR-200b/c antagomir and si-ROCK2 + si-ZEB1/2. The number of invasive cells is shown in (D). (E) and (F) Lentivirus-based sh-SUZ12transfected TFK-1 cells were used for tumour initiation. The BALB/c-nude mice were killed at 6 weeks after injection. All the data were compared with the NC group. (G) Downstream gene expression of ROCK2 after transfection with miR-200b/c mimics and antagomir.

Cofilin and thus might reduce the invasion capacity of cholangiocarcinoma cells.

To confirm the mechanism regulating the effect of SUZ12 on the tumourigenesis properties of cholangiocarcinoma, we transfected
TFK-1 cells with a lentivirus-based shRNA vector to silence the SUZ12 and ROCK2 genes (Supplementary Figure 6C) and we repeated the sphere formation and tumour-initiating assays. The SUZ12 shRNA group formed smaller spheres than the NC 
A

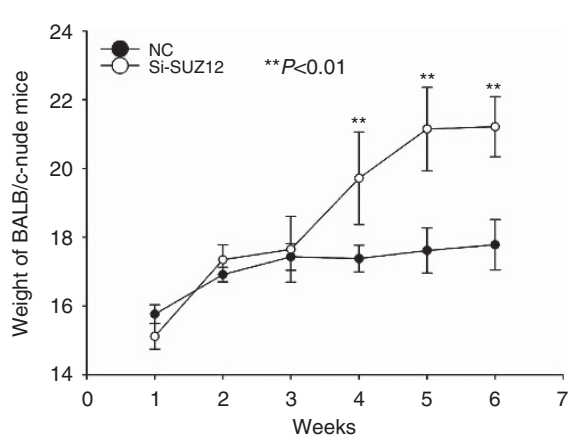

C
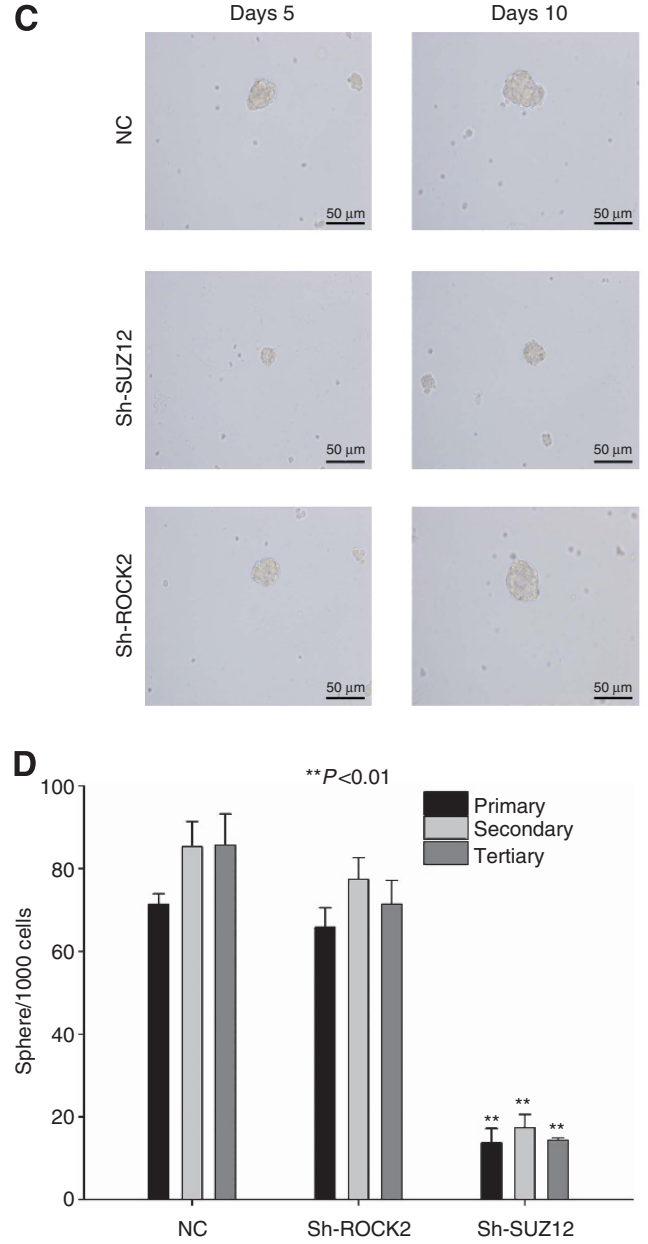

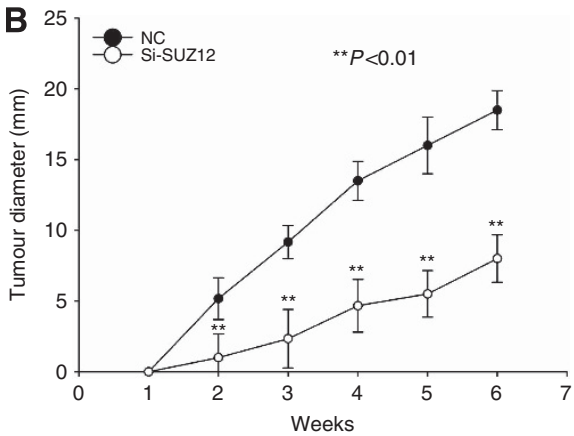

Days 15
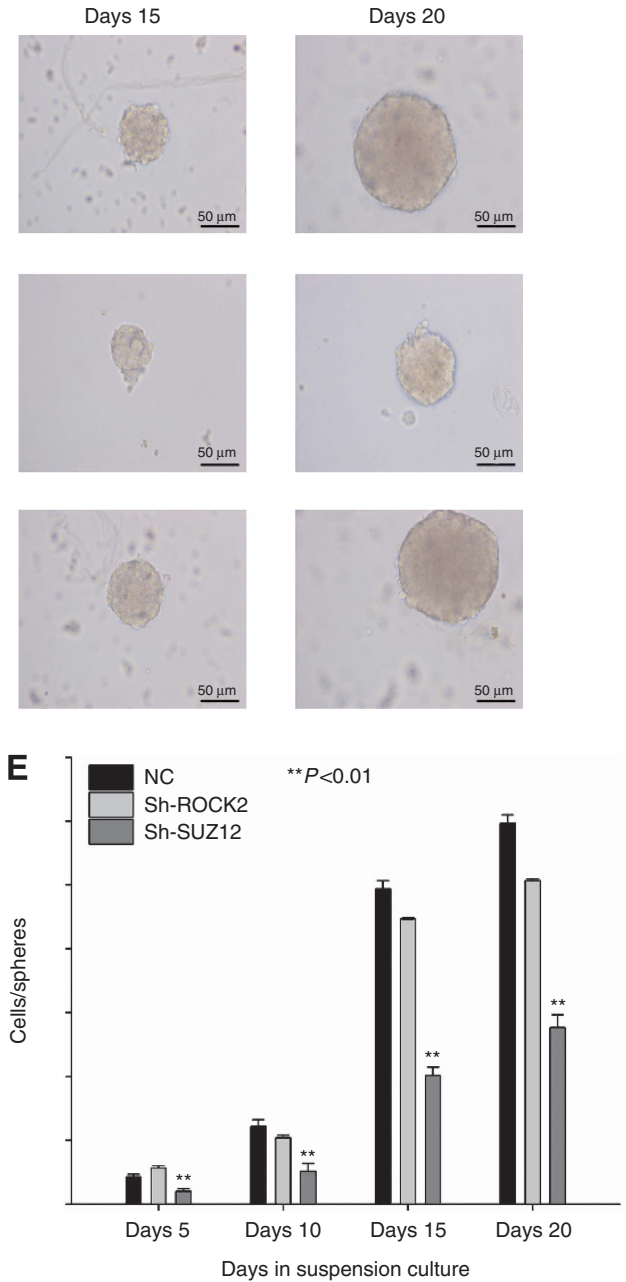

Figure 7. The weight data for the BALB/c-nude mice from the sh-SUZ12 tumour-initiating assay are shown in (A). The data for the tumour diameters are shown in (B). Lentivirus-based si-SUZ12-transfected TFK-1 cells were used for the sphere culture and differentiation assay (C). The cell per sphere values (D) and sphere per 1000 cell $(E)$ values were determined.

(Figure 7C), and the spheres of the control group increased in size and cell number more quickly than those of the SUZ12 shRNA group (Figure 7D and E). However, the sphere formation capacity of the sh-ROCK2 shRNA group showed no statistical significance compared with the NC group (Figure 7C-E). These changes were also associated with the self-renewal capacity, and the blank vector group formed tumours much more rapidly than the SUZ12-shRNA group (Figures 6E, F and 7A, B). However, the chemoresistance capacities of the SUZ12 shRNA group were not significant in comparison to the NC group. Collectively, the results indicate that SUZ12 is an important gene that is associated with some cholangiocarcinoma properties, such as sphere formation and tumour initiation, and is regulated by $\mathrm{miR}-200 \mathrm{~b} / \mathrm{c}$.

\section{DISCUSSION}

The initial studies on cholangiocarcinoma focused on its multichemoresistance and distant metastasis. To investigate the possible mechanism of the malignant biological behaviour of cholangiocarcinoma and to identify crucial regulators, we established that there was a dysregulated miRNA profile in cholangiocarcinoma tissues and found four miR-200 family members among the top six down-regulated miRNAs. Although the miR-200 family members have been demonstrated to be cancer suppressors in many forms of cancer, the function of miR-200 in cholangiocarcinoma cells has not been elucidated to date. Here, we investigated the dysregulation 
of $\mathrm{miR}-200 \mathrm{~b} / \mathrm{c}$ as a possible cause of the invasion and metastasis capacities and tumorigenic properties of cholangiocarcinoma, and we clarify a novel downstream mechanism. Overall, our results suggest a novel therapeutic strategy for cholangiocarcinoma treatment.

In comparison to normal controls, miR-200 family members are downregulated in many human cancers, including breast cancer (Teng et al, 2013), pancreatic tumours (Li et al, 2010), and hepatic cancer (Kim et al, 2011; Zhang et al, 2013). Most researchers have demonstrated that the expression of miR-200 is negatively correlated with the invasion and metastasis capacities of cancer (Hur et al, 2012; Soubani et al, 2012; Guo et al, 2013). Although the existence of the miR-200-ZEB1/2-E-cadherin axis during the EMT process was confirmed in initial studies (Teng et al, 2013), several recent studies have reported conflicting roles of miR-200 in metastatic progression. These contradictory findings may in part be due to the different source organisation and models (Korpal et al, 2011); indeed, there are different expression levels and mechanisms in different tissues. Thus, in our study, we demonstrated that $\mathrm{miR}-200 \mathrm{~b} / \mathrm{c}$ is downregulated in cholangiocarcinoma and that the knockdown of $\mathrm{miR}-200 \mathrm{~b} / \mathrm{c}$ promotes the invasion and metastasis capacities of cholangiocarcinoma cells. Similarly, we demonstrated that inhibiting the expression of ROCK2 reduces the invasion of cholangiocarcinoma cells (although the inhibition of SUZ12 had no effect). Both the overexpression of miR-200b/c and inhibition of ROCK2 can reduce the invasion of cholangiocarcinoma cells, yet ROCK2-knockdown cholangiocarcinoma cells may retain a stronger invasion capacity. The ROCK2-knockdown cholangiocarcinoma cells exhibited enhanced invasion and metastasis capacities in comparison to the $\mathrm{miR}-200 \mathrm{~b} / \mathrm{c}$-overexpression cells, indicating that the influence of miR-200 on cholangiocarcinoma invasion extends beyond the regulation of ROCK2 and most likely involves other targets. We found that the combined suppression of $Z E B 1 / 2$ and $R O C K 2$ resulted in a similar inhibition effect as that of $\mathrm{miR}-200 \mathrm{~b} / \mathrm{c}$ overexpression. Therefore, both $Z E B 1 / 2$ and ROCK2 are crucial regulatory proteins of the invasion capacity of cholangiocarcinoma cells and are silenced by miR$200 \mathrm{~b} / \mathrm{c}$.

In addition to the invasion and metastasis capacities, many researchers have demonstrated that the expression of miR-200 is associated with the stemness of cancer cells. Indeed, our experiments examining sphere formation, drug resistance, and tumour-initiating capacity addressed CSC-like properties, suggesting a possible link between miR-200b/c and cholangiocarcinoma stem-like cells. Previous studies have shown that stemness-related genes such as those of the ZEB family, which have been demonstrated to be direct targets of miR-200, consist of transcriptional repressors, including ZEB1 and ZEB2. The functions of this protein family are extensive and include development, the activation of the transforming growth factor (TGF)- $\beta$ signalling pathway in EMT, and stem cell pathways (Vandewalle et al, 2009; Sanchez-Tillo et al, 2011; Gheldof et al, 2012). According to the miR-200-ZEB dual loop theory, not only does the miR-200 family directly target $Z E B 1 / 2$ but the overexpression of $Z E B 1 / 2$ also reduces miR-200 levels. Based on the above findings, there may be two pathways by which the miR-200 family influences the stemness of cancer: (1) the suppression of $Z E B 1 / 2$, thereby influencing the stemness of CSCs via downstream proteins, or (2) the direct targeting of certain stemness-related proteins, thereby directly influencing CSC properties. There are many studies supporting the hypotheses of pathway 1, but less experimental evidence supports pathway 2. Remarkably, our results show that the protein expression level of ZEB1 in TFK-1 cells is deficient, suggesting that the proportion of $Z E B$ family related CSC-like cells in the TFK-1 cell line may be very low. Our integrated analysis of miR-200 and stemness shows that miR-200 regulates a very large number of target mRNA sequences. Thus, it appears likely that the effect of miR-200 on cholangiocarcinoma tumorigenic or stem celllike properties is not mediated solely through the miR-200-ZEB axis and that there are some target mRNAs directly regulated by miR-200. The miR-200 family members may influence the stemness of cancer via direct suppression. Our results establish that $\mathrm{miR}-200 \mathrm{~b} / \mathrm{c}$ influences the drug resistance, tumour-initiating capacity, sphere formation, and CD133 expression level, a potential CSC marker, of cholangiocarcinoma cells. SUZ12 has been reported to be a direct target of miR-200b in breast CSCs, and the direct targeting of SUZ12 by miR-200c was demonstrated in our study. The direct knockdown of SUZ12 by shRNA achieved a similar suppressive effect on the sphere formation and tumourinitiating capacities of cholangiocarcinoma as that of miR-200b/c. This result confirms that SUZ12 is important in tumourigenesis and that related genes may be directly regulated by miR-200b/c. Based on the enrichment of low-differentiation cholangiocarcinoma cells by sphere formation and drug resistance, we considered that the proportion of cholangiocarcinoma stem-like cells had been increased, and we next validated the $\mathrm{miR}-200 \mathrm{~b} / \mathrm{c}$ expression levels of the parental and differentiated spheres and drug-resistant cells. Interestingly, the expression of $\mathrm{miR}-200 \mathrm{~b} / \mathrm{c}$ was decreased during low-differentiation cell enrichment and increased upon differentiation, indicating that there may be negative feedback between miR200b/c and cholangiocarcinoma stem-like cells.

In conclusion, our findings demonstrate that miR-200b/c inhibits the invasion and metastasis capacities, and tumorigenic capacity (or the so-called CSC-like properties), including drug resistance, tumour-initiating capacity, sphere formation, and CD133 expression level, of cholangiocarcinoma cells. Such a suppression of these properties can be imitated by directly repressing ROCK2 and SUZ12, two direct targets of miR-200b/c. Thus, our observations provide very strong experimental results regarding the involvement of miR-200 in the invasion and metastasis capacities of cholangiocarcinoma, suggesting a novel therapeutic strategy for cholangiocarcinoma treatment and proposing a potential surface marker for cholangiocarcinoma stemlike cells.

\section{ACKNOWLEDGEMENTS}

This study was funded by The National Natural Science Foundation of China (nos. 81071775 and 81272659) to RQ, no. 81101621 to MW, no. 81160311 to JJ, no. 81172064 to MS, no. 81001068 to RT, no. 81272425 to XW, and no. 81101870 to Jun $\mathrm{Hu}$; National 'Eleventh Five-Year' Scientific and Technological Support Projects (no. 2006BAI02A13-402) to RQ; Key Projects of Science Foundation of Hubei Province (no. 2011CDA030) to RQ; and Research Fund of Young Scholars for the Doctoral Program of Higher Education of China (no. 20110142120014) to MW.

\section{REFERENCES}

Almontashiri NA, Fan M, Cheng BL, Chen HH, Roberts R, Stewart AF (2013) Interferon-gamma activates expression of p15 and p16 regardless of 9p21.3 coronary artery disease risk genotype. J Am Coll Cardiol 61(2): 143-147.

Blechacz B, Gores GJ (2008) Cholangiocarcinoma: advances in pathogenesis, diagnosis, and treatment. Hepatology 48(1): 308-321.

Burk U, Schubert J, Wellner U, Schmalhofer O, Vincan E, Spaderna S, Brabletz T (2008) A reciprocal repression between ZEB1 and members of the miR-200 family promotes EMT and invasion in cancer cells. EMBO Rep 9(6): 582-589.

Cochrane DR, Howe EN, Spoelstra NS, Richer JK (2010) Loss of miR-200c: a marker of aggressiveness and chemoresistance in female reproductive cancers. J Oncol 2010: 821717. 
Dykxhoorn DM, Wu Y, Xie H, Yu F, Lal A, Petrocca F, Martinvalet D, Song E, Lim B, Lieberman J (2009) miR-200 enhances mouse breast cancer cell colonization to form distant metastases. PLoS One 4(9): e7181.

Evert M, Dombrowski F, Biao F, Ribback S, Chen X, Calvisi DF (2013) On the role of Notch1 and adult hepatocytes in murine intrahepatic cholangiocarcinoma development. Hepatology; e-pub ahead of print 23 March 2013; doi:10.1002/hep.26411.

Gheldof A, Hulpiau P, van Roy F, De Craene B, Berx G (2012) Evolutionary functional analysis and molecular regulation of the ZEB transcription factors. Cell Mol Life Sci 69(15): 2527-2541.

Guo L, Chen C, Shi M, Wang F, Chen X, Diao D, Hu M, Yu M, Qian L, Guo N (2013) Stat3-coordinated Lin-28-let-7-HMGA2 and miR-200-ZEB1 circuits initiate and maintain oncostatin M-driven epithelial-mesenchymal transition. Oncogene; e-pub ahead of print 14 January 2013; doi:10.1038/ onc. 2012.573

He L, Hannon GJ (2004) MicroRNAs: small RNAs with a big role in gene regulation. Nat Rev Genet 5(7): 522-531.

Hermann PC, Huber SL, Herrler T, Aicher A, Ellwart JW, Guba M, Bruns CJ, Heeschen C (2007) Distinct populations of cancer stem cells determine tumor growth and metastatic activity in human pancreatic cancer. Cell Stem Cell 1(3): 313-323.

Hur K, Toiyama Y, Takahashi M, Balaguer F, Nagasaka T, Koike J, Hemmi H, Koi M, Boland CR, Goel A (2012) MicroRNA-200c modulates epithelialto-mesenchymal transition (EMT) in human colorectal cancer metastasis. Gut 62(9): 1315-1326.

Iliopoulos D, Lindahl-Allen M, Polytarchou C, Hirsch HA, Tsichlis PN, Struhl K (2010) Loss of miR-200 inhibition of Suz12 leads to polycomb-mediated repression required for the formation and maintenance of cancer stem cells. Mol cell 39(5): 761-772.

Karres JS, Hilgers V, Carrera I, Treisman J, Cohen SM (2007) The conserved microRNA miR-8 tunes atrophin levels to prevent neurodegeneration in Drosophila. Cell 131(1): 136-145.

Khan SA, Thomas HC, Davidson BR, Taylor-Robinson SD (2005) Cholangiocarcinoma. Lancet 366(9493): 1303-1314.

Khan SA, Toledano MB, Taylor-Robinson SD (2008) Epidemiology, risk factors, and pathogenesis of cholangiocarcinoma. Off J Int Hepato Pancreato Biliary Assoc 10(2): 77-82.

Kim T, Veronese A, Pichiorri F, Lee TJ, Jeon YJ, Volinia S, Pineau P, Marchio A, Palatini J, Suh SS, Alder H, Liu CG, Dejean A, Croce CM (2011) p53 regulates epithelial-mesenchymal transition through microRNAs targeting ZEB1 and ZEB2. J Exp Med 208(5): 875-883.

Korpal M, Ell BJ, Buffa FM, Ibrahim T, Blanco MA, Celia-Terrassa T, Mercatali L, Khan Z, Goodarzi H, Hua Y, Wei Y, Hu G, Garcia BA, Ragoussis J, Amadori D, Harris AL, Kang Y (2011) Direct targeting of Sec23a by miR-200s influences cancer cell secretome and promotes metastatic colonization. Nat Med 17(9): 1101-1108.

Korpal M, Lee ES, Hu G, Kang Y (2008) The miR-200 family inhibits epithelial-mesenchymal transition and cancer cell migration by direct targeting of E-cadherin transcriptional repressors ZEB1 and ZEB2. J Biol Chem 283(22): 14910-14914.

Li A, Omura N, Hong SM, Vincent A, Walter K, Griffith M, Borges M, Goggins M (2010) Pancreatic cancers epigenetically silence SIP1 and hypomethylate and overexpress miR-200a/200b in association with elevated circulating miR-200a and miR-200b levels. Cancer Res 70(13): 5226-5237.

Park SM, Gaur AB, Lengyel E, Peter ME (2008) The miR-200 family determines the epithelial phenotype of cancer cells by targeting the E-cadherin repressors ZEB1 and ZEB2. Genes Dev 22(7): 894-907.
Samavarchi-Tehrani P, Golipour A, David L, Sung HK, Beyer TA, Datti A, Woltjen K, Nagy A, Wrana JL (2010) Functional genomics reveals a BMPdriven mesenchymal-to-epithelial transition in the initiation of somatic cell reprogramming. Cell Stem Cell 7(1): 64-77.

Sanchez-Tillo E, Siles L, de Barrios O, Cuatrecasas M, Vaquero EC, Castells A, Postigo A (2011) Expanding roles of ZEB factors in tumorigenesis and tumor progression. Am J Cancer Res 1(7): 897-912.

Schickel R, Park SM, Murmann AE, Peter ME (2010) miR-200c regulates induction of apoptosis through CD95 by targeting FAP-1. Mol Cell 38(6): 908-915.

Shimono Y, Zabala M, Cho RW, Lobo N, Dalerba P, Qian D, Diehn M, Liu H, Panula SP, Chiao E, Dirbas FM, Somlo G, Pera RA, Lao K, Clarke MF (2009) Downregulation of miRNA-200c links breast cancer stem cells with normal stem cells. Cell 138(3): 592-603.

Soubani O, Ali AS, Logna F, Ali S, Philip PA, Sarkar FH (2012) Re-expression of miR-200 by novel approaches regulates the expression of PTEN and MT1-MMP in pancreatic cancer. Carcinogenesis 33(8): 1563-1571.

Teng Y, Mei Y, Hawthorn L, Cowell JK (2013) WASF3 regulates miR-200 inactivation by ZEB1 through suppression of KISS1 leading to increased invasiveness in breast cancer cells. Oncogene. e-pub ahead of print January 14 2013; doi:10.1038/onc.2012.565.

Timmer MR, Beuers U, Fockens P, Ponsioen CY, Rauws EA, Wang KK, Krishnadath KK (2013) Genetic and epigenetic abnormalities in primary sclerosing cholangitis-associated cholangiocarcinoma. Inflamm Bowel Dis 19(8): 1789-1797.

Vandewalle C, Van Roy F, Berx G (2009) The role of the ZEB family of transcription factors in development and disease. Cell Mol Life Sci 66(5): 773-787.

Visvader JE, Lindeman GJ (2008) Cancer stem cells in solid tumours: accumulating evidence and unresolved questions. Nat Rev Cancer 8(10): $755-768$.

Wang M, Xiao J, Jiang J, Qin R (2011a) CD133 and ALDH may be the molecular markers of cholangiocarcinoma stem cells. Int J Cancer 128(8): 1996-1997.

Wang M, Xiao J, Shen M, Yahong Y, Tian R, Zhu F, Jiang J, Du Z, Hu J, Liu W, Qin R (2011b) Isolation and characterization of tumorigenic extrahepatic cholangiocarcinoma cells with stem cell-like properties. Int J Cancer 128(1): 72-81.

Wellner U, Schubert J, Burk UC, Schmalhofer O, Zhu F, Sonntag A, Waldvogel B, Vannier C, Darling D, zur Hausen A, Brunton VG, Morton J, Sansom O, Schuler J, Stemmler MP, Herzberger C, Hopt U, Keck T, Brabletz S, Brabletz T (2009) The EMT-activator ZEB1 promotes tumorigenicity by repressing stemness-inhibiting microRNAs. Nat Cell Biol 11(12): 1487-1495.

Yu F, Yao H, Zhu P, Zhang X, Pan Q, Gong C, Huang Y, Hu X, Su F, Lieberman J, Song E (2007) let-7 regulates self renewal and tumorigenicity of breast cancer cells. Cell 131(6): 1109-1123.

Zhang L, Yang F, Yuan JH, Yuan SX, Zhou WP, Huo XS, Xu D, Bi HS, Wang F, Sun SH (2013) Epigenetic activation of the MiR-200 family contributes to H19-mediated metastasis suppression in hepatocellular carcinoma. Carcinogenesis 34(3): 577-586.

This work is published under the standard license to publish agreement. After 12 months the work will become freely available and the license terms will switch to a Creative Commons AttributionNonCommercial-Share Alike 3.0 Unported License.

Supplementary Information accompanies this paper on British Journal of Cancer website (http://www.nature.com/bjc) 